\title{
Research on Training circulation Based on Employee Personas
}

\author{
Xuming Lou ${ }^{1, a}$, Ya'nan Zhao ${ }^{1, b, *}$, Sen Yang ${ }^{2, c}$ \\ ${ }^{1}$ I School of Business and Management, Xi'an University of Posts and Telecommunications, Xi'an \\ 710000, China; \\ 22Department of HRM, OCT Xi'an Industrial Co., Ltd., Xi’an 710000, China. \\ alouxuming@xupt.edu.cn, bava_zyn@126.com, caqh1989@163.com
}

Keywords: Training system, Employee personas, PDCA cycle.

\begin{abstract}
Based on the continuous improvement characteristics of PDCA cycle, this paper preliminarily constructs the training circulatory for enterprise employees. In accordance with the four phases of the PDCA cycle, four modules including training planning, training implementation, training evaluation, and training improvement were set up for the system. At the same time, in order to achieve the precise goal of enterprise training, the employee personas database was established and employee personas were applied to the training circulatory to improve the quality of staff training.
\end{abstract}

\section{Introduction}

To improve the ability to continually improve the quality and the effectiveness of training, this paper borrowed the concept of PDCA cycle to construct a training circulatory. Meanwhile, to ensure that the training is more in line with the real needs of employees, this paper applies the portrait of the employees to the implementation of the training cycle to improve the accuracy of training and meet the requirements of personalized management .

\section{PDCA cycle}

\subsection{The meaning of PDCA cycle}

PDCA cycle was first out Walter A. Shewhart conceived in 1930, it was given a "plan-Do-See" mode. Afterwards, W.Edwards Deming, a master of quality control, improved the PDS mode of Shewhart in 1950 and further developed the PDCA cycle mode - Plan, Do, Check, Action. This was widely publicized and the new PDCA cycle model was applied to the continuous improvement of product quality[1].Through the PDCA cycle, companies can find and improve a variety of management problems. At the same time, PDCA cycle can also be applied to various fields, such as large enterprise strategic management, government environmental pollution control, small to departmental project management, staff training and self-management. PDCA cycle is continuously, formally or informally, consciously or subconsciously used in everything people has done.

\subsection{Four phases of PDCA cycle}

PDCA cycle usually consists of four phases, namely P-planning phase, D-doing phase, $\mathrm{C}$-checking phase, and A-action phase. Phase P-This is the planning phase. This phase is to conduct a comprehensive analysis of the problems or opportunities that arise, study and analyze it from as many angles as possible, find its root cause, and design one or more corrections to the problem or seize this opportunity and develop an implementation plan. Phase D-implementation phase, according to the plan formulated in Phase $\mathrm{P}$, design and layout scheme, and carry out specific operations to maximize the expected goals. Phase C-In this phase, it is necessary to evaluate the effectiveness of the implementation of the plan, these effects will be compared with the stated objectives or expected results. Then, confirm whether the target is achieved, that is, check, verify, and evaluate the effect. Phase A -In order to perform and promotion, the new processes, systems or solutions will be identified as the standard processes, solutions or production methods, if the results of the inspection was satisfactory; instead, you need to take corrective action. 


\section{Preliminary construction of the training circulatory}

\subsection{The construction concept of training circulatory}

The training circulatory applies the concept of PDCA cycle to corporate training management work. According to the process of training work, it is divided into four steps: training planning, training implementation, training evaluation and training improvement, so that it can correspond to the four phases of the PDCA cycle (as shown in Figure 1) [2].

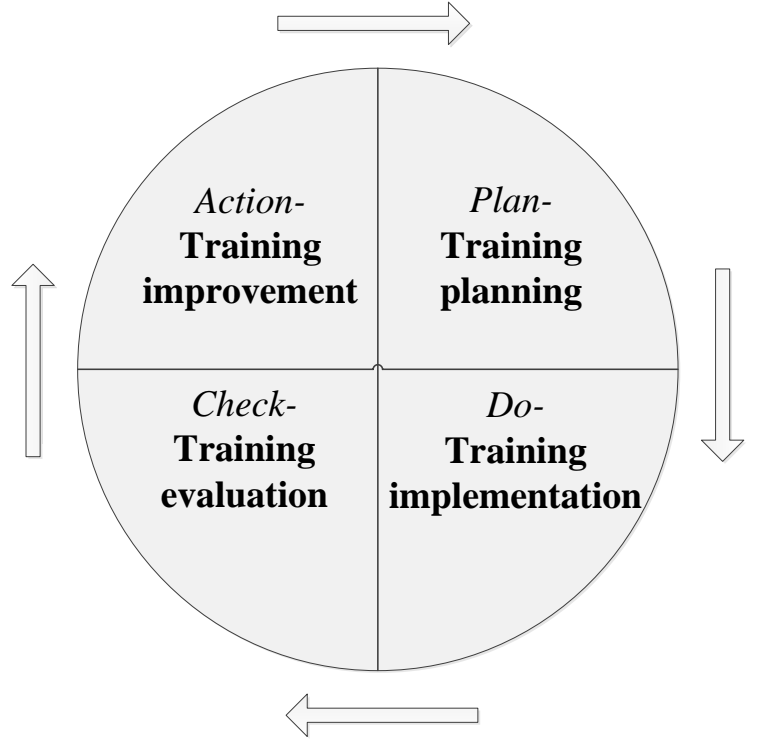

Fig. 1 Training cycle diagram

Phase P corresponds to training planning. The work in this phase mainly includes the analysis of employees training needs and the determination of training programs. Phase $\mathrm{D}$ corresponds to training implementation. The training plan identified in Phase P is implemented and employees are trained. Which corresponds to phase $\mathrm{C}$ is training evaluation, that is, evaluate the effectiveness of the training, check whether the training program is reasonable, whether the training content meets the needs of employees, and whether employees meet the training goals. Phase $\mathrm{A}$ is training improvement phase. It summarizes experiences for training evaluations, consolidates results, and transfers problems in the cycle to the next training cycle.

\subsection{Training planning}

Training planning refers to the analysis of training needs based on the company's development strategy, and the combination of the employees' skill level and post competency requirements.

Analysis of training needs. The premise of the company's development of the training plan and the identification of the training plan is the analysis of the training needs. The training plan can be reflected in the training program and is also inseparable from the analysis of training needs. According to Goldstein's three-level model, training needs analysis should be based on the training plan, and from the three levels of organization, tasks, and personnel[3].Training needs analysis should be based on corporate development strategies and training programs. Combining job competency requirements and knowledge ability standards, analyzing the gap between the actual work performance of employees and the acceptable performance standards of the job and the reasons (current training needs), the gap between the proficiency of employees in each technology and the required proficiency of the technology and the reasons for this (future training needs).

Determine the training program. After training needs are determined, Based on the specific requirements of the company, trained employees and training departments, by using the $5 \mathrm{~W} 1 \mathrm{H}$ analysis[4], clear definition of training content (What), training goal (Why), training lecturer (Who), training time (When), training location (Where), training form (How), determine a reasonable training goal, complete the specific content of the training plan, etc., and transform training needs into training programs[5]. 


\subsection{Training implementation}

In order to ensure the normal implementation of the training program, training departments as training organizers should take the necessary safeguard measures, including the identification of training instructors, training topics and choice of training forms, as well as training environment and training, after completing the analysis of training needs and forming a training program.

At the same time, establish a communication feedback mechanism in the training process to ensure the circulation of information, continue to summarize, and In order to ensure the circulation of information, a communication feedback mechanism in the training process is established.at the same time, it is necessary to continuously summarize and timely solve the problems arising in the training process.

\subsection{Training evaluation}

Donald L Kirkpatrick proposed the Kirkpatrick Model in 1959 [6]. It is the most widely used training assessment tool in the world. There are four levels in the model-a response layer, a learning layer, a behavior layer and a effectiveness layer, the four levels are progressive.

Training evaluation mainly starts from the following four aspects: (1) To evaluate the through satisfaction survey on effectiveness of training according to the employees' subjective feelings on training contents, training instructors, training forms, training environment, etc.. This is about the evaluation of the reaction layer.(2) Evaluate the training of trainees in the training content by the written test, skill operation or scenario simulation, etc. to understand the learning effect of employees, this is about the evaluation of the learning layer.(3) Behavioral layer: After employees training, they can judge whether the training can change the work behavior of the employees and whether the work ability is improved by observing the employees' learning and work performance. This is the third layer of behavioral evaluation.(4) The fourth layer is the evaluation of the benefit layer, and the key point is to judge whether its performance has been improved based on the employee's behavior after receiving the training, and to evaluate the benefits of changes in their behavior to individuals and enterprises.

\subsection{Training improvement}

The training improvement is analyzed on the basis of the evaluation of the training effect and takes corresponding measures according to the specific situation. The successful experience form is standardized and applied to the subsequent training; the problems found during the training process are summarized, and corresponding strategies are formulated to improve and avoid secondary mistakes and achieve continuous improvement of training.

The completion of the training improvement work represents the end of a training cycle. However, in the training cycle, training improvement does not mean that the training work is over. The next PDCA cycle should be improved according to the improvement measures and the next training cycle should be started. The training plan of the next PDCA cycle, based on the training improvement strategy formulated in the above training cycle, will help improve the quality of training, improve the training system, and achieve continuous development of training.

\section{Employee personas}

\subsection{The meaning of employee personas}

At present, more and more companies have set up an E-Learning platform. The fragmented behavior trajectories of employees such as browsing, clicking, commenting, and commenting on the platform have been collected and stored[7]. These fragmented, collated data stored in the database can be used to analyze and mine employees' needs and preferences, and is the decision-making basis for companies to implement employee-specific management and precise training. These all-round, three-dimensional records and depictions of employee data, include all employees' personal basic information, online behavior information, and employees' offline behavioral information (work behavior performance, performance output, assessment results, etc.), are called “employee personas”.

A tag is usually a highly-refined feature tag artificially specified, such as gender, age, education, job position, skill level, employee preferences, etc. The main job of employee personas is to label the 
employee "tags" and then to summarize all the tags, and then sketched out employee "personas"[8].The employee portrait mainly includes three steps. The first step is to obtain the original data, which mainly includes the basic attribute information of employees and the behavior information from the E-learning platform. This part mainly analyzes the behavior data of employees through data mining techniques. The second step is to count and analyze the original data and obtain the labels needed for portraits, such as gender, age, education, position, skill level, employee preference, etc.. The third step is to further analyze the obtained tags to summarize the final tags, such as the basic attribute characteristics of employees and online behavior preferences. Then, the employees are grouped by the attributes of the obtained tags and their behaviors are predicted.

In the era of big data, the "employees personas" composed of data not only reproduced the overall image of employees as training objects, but also reflected their real needs. It is also the foundation for the company to tap out employees' training needs, conduct segment employees, and achieve accurate training.

\subsection{Employees personas database}

The training circulatory based on employee personas is to build a database of employee personas, collect data from employee personas database, and view employees' feedback data as materials for continuously updating and improving the training system.

Constructing a employee personas database, it can be used as data mining objects with basic information and behavior information of employee (as shown in Figure 2).Employees' behavior information includes online behavior information and offline behavior information. The corresponding mining tasks include the identification of employees' browsing behaviors, the online interactive behavior analysis of employee comments, likes and comments, and the analysis of employees' online learning self-evaluation results. Offline behavior information refers to the employee's information in behavior and performance, including employee's daily attendance, other people's evaluation, performance output, the gap between current performance and standard performance, and the gap between current skill proficiency and skills mastery standards.

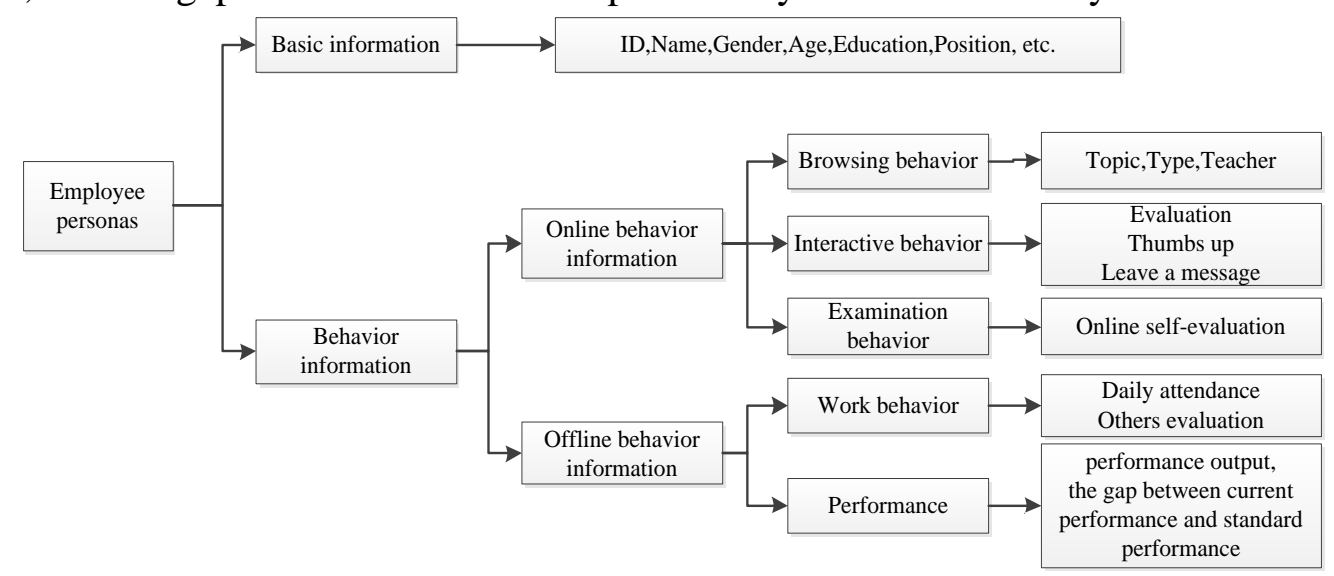

Figure 2 Employee personas

\section{Training circulatory based on employee personas}

\subsection{Construction ideas}

Training circulatory based on employee personas is constantly collecting and tracking changes in corporate strategy, business activities, personnel changes, and management systems and processes changes during the cyclical implementation of the training work on the basis of employee personas, so that we can timely grasp the current needs of employees, and correctly predict the future training needs of employees. Training circulatory aims to provide continuous feedback on the training of employees, to analyze training needs on a regular basis[9], to supervise the implementation of training, and to formulate successful experiences on the basis of training evaluations.

This article adds the function of employee personas during the training cycle. As shown in Figure 3 , employee personas provide internal power during the entire cycle of the training work, and 
continuously promote the quality of the training. Just like the transmission of the vehicle accelerates the cycle and constitutes a mode similar to the large ring in the PDCA cycle, rolling forward, cycling, and spiraling up.

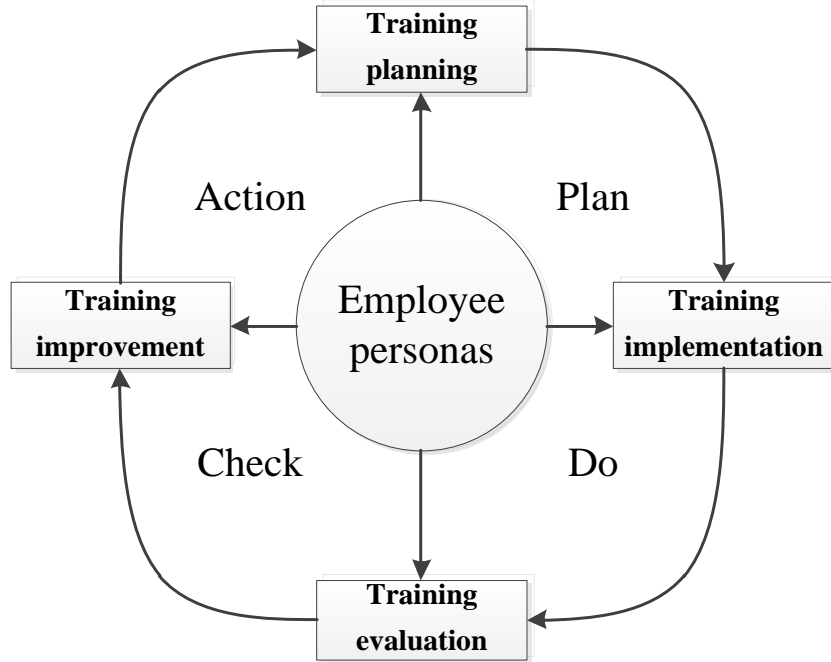

Figure 3 Training circulatory based on employee personas

\subsection{Employee personas and training needs analysis}

Based on the training circulatory based on employee personas, the $5 \mathrm{~W} 1 \mathrm{H}$ analysis method was expanded to $6 \mathrm{~W} 1 \mathrm{H}$, which increased the analysis items of the trainee (Whom). The role of employee personas in the training planning stage was mainly reflected in the analysis of employees' training needs. At this stage, the main contribution of employee personas is to make up for the previous training model that only focused on employee performance and skills while ignoring the interest and preference of employees. While valuing the actual needs of employees, it also considered the psychological appeals of employees, and fully integrate "what employees must learn" and "what they want to learn”.

Based on the information provided by the database of employee personas, collect information on demand attributes that reflect the actual needs and preferences of employees. Online browsing behaviors and interaction behaviors reflect employees' psychological appeals. The topics, types, and lecturers of the content that employees browsing, the length of time, number of likes, and number of comments made by employees on the page are all true reflections of employees' interests and preferences. The offline performance information and online self-evaluation results, such as employees' performance and assessment results, reflect the employee's position in terms of competency.

After obtaining information about the employees' demand attributes, employees are grouped according to the demand attributes through data mining algorithms such as association rules, clustering, classification, and regression[10].Finally, corresponding training programs are formed according to different requirements attributes (as shown in Figure 4). 


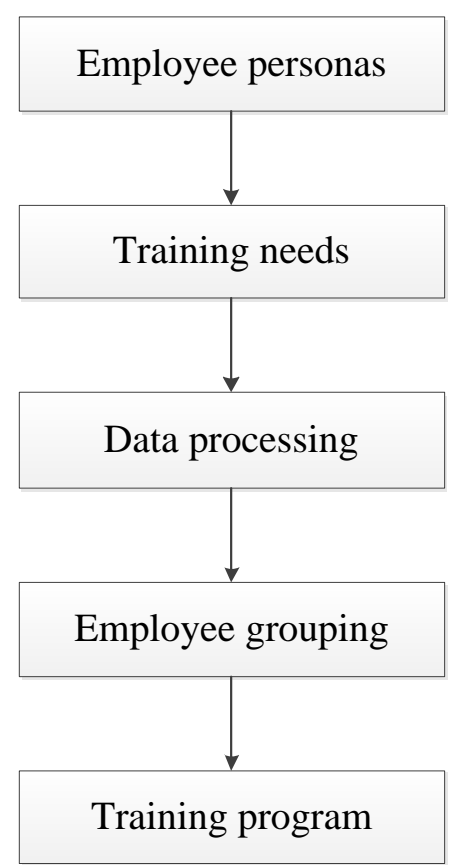

Figure 4 Application of employee personas in training planning phrase

\subsection{Employee personas and training implementation}

In the implementation phase of the training, the main purpose of the employee personas is to keep track of employees' perceptions of the training work. Including employees' perceptions and evaluations of training content, training forms, training instructors, etc., as well as employees' knowledge of training content.

The work of employee personas during the training implementation phase and are similar to information feedback systems. In the process of receiving training, employees will continue to convey information on training satisfaction, such as subjective feelings about training instructors, training content, training forms, training environment and time. Training instructors play an important role in guiding and inspiring the entire training process. They should select the right instructor according to the trainees to meet the actual situation of the training. The training content and training form should be in line with the skills level, academic education, and work of the trainee. The training environment needs to be selected in conjunction with the rank and qualifications of the trainees. Training instructors should also vary from person to person in the arrangement of training time, and should avoid working hours of the trainees, and the duration of a class should also be controlled.

\subsection{Employee personas and training evaluation}

There are two aspects of employee personas in the training evaluation phase: on the one hand, the evaluation on the training effect is based on what the employee wants to learn, and on the other hand, the training evaluation is based on what the employee should or must learn. The effectiveness evaluation of "what employees want to learn" can be achieved through satisfaction surveys. The survey content should include the subjective feelings of employees on training contents, training instructors, training forms, training environment and time. To evaluate the effectiveness of "what employees should or must learn”, we can understand employees' learning effects through the written test, skill operation or scenario simulation, etc. Similarly, by observing the learning and work performance of employees, we can judge whether the training can change and the benefits generated by the company.

\subsection{Employee personas and training improvement}

After judging the improvement in the level of skills and performance and evaluating the changes in their behavior, training organizers must summarize and analyze this training work, find and deal with problems, summarize experiences, and consolidate achievements.

In the training improvement phase, based on the results data from the training evaluation phase, a new persona of employees is formed and the new employee personas is compared with the old one 
which formed before the training. Analyze the success and failure of training, identify successful experience and standardize it, and apply it to subsequent training, from the changes in employee skills and performance output as well as the degree of satisfaction of employees' hobbies , and analyze the problems that arise and identify the causes and make improvements.

Review and analyze in the stage of training improvement, and summarize the opinions of related parties, form a training implementation report, put forward the successful aspects of training and areas for improvement, and then enter a new round of training work.

\section{Conclusions}

Any training is carried out purposefully. The end of one training is the beginning of another training and can be used as input for the next training plan. As a systematic training circulatory, the four aspects of training planning, training implementation, training evaluation, and training improvement are related to each other and influence each other. Only by systematically organizing and implementing training can we achieve the desired results.

In each training cycle, the analysis needs to be conducted from the overall level of the enterprise, the job level and the individual level of employees. However, in the process of training needs analysis, Goldstein's three-level model focuses on the gap between employees' current performance and skill mastery and ideal level, and focuses on what the employee "should or must learn", ignores "what employees want to learn". Employee personas make up for this deficiency. On the one hand, employee personas should consider the expansion and extension of employees' breadth and depth of knowledge, and pay attention to the improvement of employees' skill level and performance level. At the same time, employees persona are in line with the concept of human-based training in corporate training. In consideration of employees' psychological appeal, employees' satisfaction is continuously improved by satisfying employees’ interests and hobbies.

\section{Acknowledgments}

This work was financially supported by Xi'an University of Posts and Telecommunications innovation fund.

*Corresponding author. Tel: 18392019369; E-mail:ava_zyn@126.com

\section{References}

[1] D.K. Sobek, A. Smolly, Understanding A3 Thinking: A Critical Component of Toyota's PDCA Management.2008.

[2] National Bureau of Quality and Technical Supervision. GB/T19025-2001 Quality Management Training Guide,Beijing,2004

[3] Hu Jianping,Study on Training of Rural Human Resources Based on Goldstein Model, Journal of Anhui Agricultural Sciences,2011,39(01):554-555+559.

[4] Yuan Wei, Liu Lu, Construction of the Training System in High-tech Enterprises Based on PDCA, Electronic Science and Technology, 2013, 26(08): 188-189.

[5] Li Limei, Wu Xinnian. Analysis of Evolution of Industrial Competitive Intelligence Service Model[J]. Library and Information Service, 2016,60(23):37-45.

[6] D. Kirkpatrick,Great ideas revisited: Techniques for Evaluating Training Programs, Training \& Development, 1996: 54-59

[7] Liu Hai, Lu Hui, Jin Jinhua, et al. Research on Precision Marketing Segmentation Model Based on Mining "Persona" ,Silk,2015,52(12):37-42+47. 
[8] LIU S,The Persona in Digital Library Knowledge Discovery System — Taking Tianjin Library as an Example,Library Theory and Practice, 2017(06):103-106.

[9] Wang Mingji. General Plan of Demand Analysis on Public Servant Training,Journal of Tianjin Administration Institute , 2005(03):40-43.

[10] Zhang Liangjun,Data Mining: Practical Case Analysis,Beijing, 2013,PP.24-40. 